\title{
Mathematical modelling on the control of HIV/AIDS with campaign on vaccination and therapy
}

\author{
David Omale ${ }^{1, *}$ \\ ${ }^{1}$ Mathematical Sciences Department, Faculty of Natural Sciences, Kogi State University, Anyigba, Kogi \\ State, Nigeria.
}

\begin{abstract}
HIV/AIDS is a global pandemic ravaging nations with negative adverse effect creating high level of mortality rate and increase the number of orphans worldwide. The control of this pandemic disease like any other diseases becomes a global fight, thus we use mathematical epidemic model with effective public health campaign on the need for vaccination and effective therapeutic dose as a measure to it spread. A Susceptible, Vaccinated, Infected, Treatment and AIDS (S VITA) compartmental model is developed in the study. We conducted a qualitative analysis on the models by carrying out the local and global stability analysis on the disease free and endemic equilibrium point. The basic reproduction number $R_{0}$ is found using the next generation matrix approach. The condition $R_{0}<1$ indicates that with effective public health campaign on the need for vaccination and therapeutic dose, the HIV/AIDS burden will be drastically reduced together with the transmission rate of HIV/AIDS within the population.
\end{abstract}

\section{Introduction}

Human immunodeficiency virus infection and acquired immune deficiency syndrome HIV/AIDS is a spectrum of conditions caused by infection with the human immunodeficiency virus HIV [1]. Since the discovery of HIV/AIDS the disease had caused a lot of havoc in the world, most especially in Africa and some less develop countries. HIV is transmitted by three main routes: Sexual contacts, exposure to infected body fluids or tissues and from mother to child during pregnancy delivery or breast feeding(Vertical transmission [2]). HIV/AIDS can be prevented through safe sex, needle exchange programmes, treating those who are infected and male circumcision [3]. HIV is believed to have originated in West Central Africa during the late 19th or 20th century [5]. AIDS was first recognised by the United State Centre for Disease Control and Prevention CDC in 1981 Gallo (2006). Since its inception it has caused an estimated 35 million death worldwide and it is consider a pandemic disease [4].

Vaccine aims to induce an immune response against HIV in which antibodies against HIV are administered [6]. There are no licensed HIV/AIDS vaccine on the market currently, but there is hope that the vaccine will be possible soon, because one HIV vaccine regime RV 144 has been shown to prevent HIV in some individual in Thailand.

Highly Active Antiretroviral Therapy (HAART) decreases the patient's total burden of HIV, burst the immune system and prevent any onset of opportunistic infections that often lead to death. HAART also prevent the transmission of HIV/AIDS (Eisinger et al (2019)).

\footnotetext{
*e-mail: davidubahi@gmail.com
} 
Public health education to the public had become the main strategies to curb the spread of this pandemic disease. Campaign on the need for vaccination on the part of those who are currently tested negative to HIV virus and therapeutic dose to those tested positive will go a long way to halt the progressive rate of HIV/AIDS infection globally. Owen et al (1998) used a single risk group model of the epidemic among gay men in San Francisco to examine the effects of prophylactic and therapeutic vaccines [9]. Blower et al (2002) uses a detailed model of vaccination response to investigate the effects of a number of vaccine characteristics on impact [10]. Gray et al (2003) analyzed the effect of ART and vaccination on HIV transmission using data from a cohort study in Rokai, Uganda. Mudandavire (2009) proposed a system of discreate time delay differential equation in modelling effects of public health education campaign on HIV/AIDS transmission with no other intervention available [11]. This work differs from all others in the sense that we consider the effect of public health campaign on vaccination for the active age group who are not yet positive and education on the HAART consistency on those infectious group within the population, using a deterministic differential models Ratera et al (2012), study the effect of screening and treatment on transmission of HIV/AIDS infection in a population, which is quite different from the present approach, they show that screening the unaware HIV infective and treatment of screened HIV infective have the effect of reducing the rate of transmission of HIV disease. Gumel et al (2002) present a mathematical model to design and monitor the interaction between HIV, CD4+ TCells, CD8+ T-Cell productively infected and latently infected CD4+ T-Cell and to evaluate therapeutic strategies during the early state of HIV/AIDS infection within the cell [13].

\section{Model description and analysis}

The model is based on the following assumptions:

1. The population under consideration is homogeneous

2. In the population, there is no vertical transmission, the population consist of active sex adult.

3. And that those in the active aids group doesn't transmit the virus because they may be too ill to be involved in active sex.

4. That vaccine is effective

The model is a deterministic epidemic models with the general population $N$ consist of $N=$ $S+V+I+T+A$ classes, where $\mathrm{S}$ is the susceptible, $\mathrm{V}$ the vaccination class, I represent the infectious class, $T$ is the treatment class of the infectious and A represents the active AIDS class. The population is being recruited by the parameter $\Lambda$ into the susceptible population, and through aggressive public education, the susceptible population go for vaccination at the $\left(1+w_{1}\right)$. The susceptible will be infected with the force of infection $\psi=\frac{\left(C_{1} \beta_{1}+C_{2} \beta_{2}\right) S}{N}$ into the infectious and treatment class respectively, $\gamma$ represent the rate that infected individual developed a full blown AIDS and $(1-\gamma)$ represent the rate that some proportion of the infected class under treatment with the public health campaigns $w_{2} . \sigma$ represent treatment failure which may caused those in the infected class to developed full blown AIDS. The AIDS class received treatment at the rate $\theta$. All the classes is being reduced by natural death rate denoted by $\mu$ and $\alpha$ is the diseases induced death rate. $\left(1+w_{2}\right)$ represents public health campaign for the infected class to undergo treatment and $\left(1+w_{1}\right)$ represents the public health campaign on vaccination for the susceptible class and $\phi$ is the wanning rate of vaccination.

Consider the model flow diagram below 


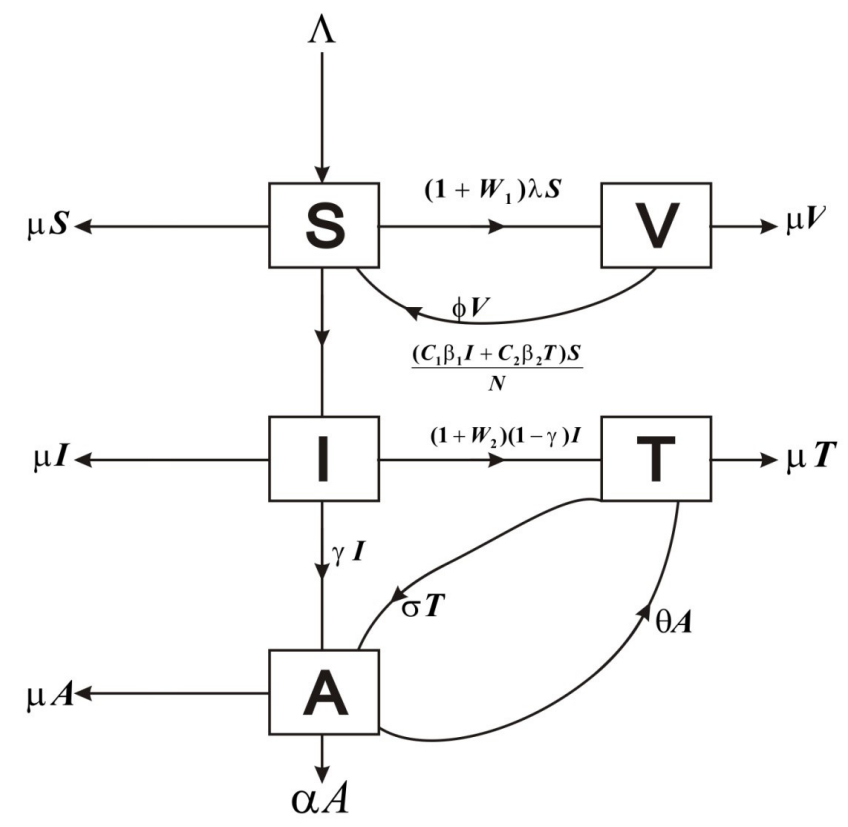

Figure 1. Model Diagram

The mathematical model arising from the above schematic diagram is:

$$
\begin{aligned}
& \frac{d S}{d t}=\Lambda-\left(1+w_{1}\right) \lambda S-\left(\frac{C_{1} B_{1} I+C_{2} B_{2} T}{N}\right) S+\phi V-\mu S . \\
& \frac{d V}{d t}=\left(1+w_{1}\right) \lambda S-\phi V-\mu V . \\
& \frac{d I}{d t}=\left(\frac{C_{1} B_{1} I+C_{2} B_{2} T}{N}\right) S-\left(1+w_{2}\right)(1-\gamma) I-\gamma I-\mu I . \\
& \frac{d T}{d t}=\left(1+w_{2}\right)(1-\gamma) I+\theta A-\sigma T-\mu T . \\
& \frac{d A}{d t}=\gamma I+\sigma T-\theta A-\alpha A-\mu A .
\end{aligned}
$$

Therefore, the disease free equilibrium point is given as

$$
\varepsilon_{0}=\left(\frac{\Lambda}{\left[\left(1+w_{1}\right) \lambda+\mu-\frac{\phi}{(\phi+\mu)}\left(1+w_{1}\right) \lambda\right]}, \frac{\left(1+w_{1}\right) \lambda \Lambda}{[\phi+\mu]\left[\left(1+w_{1}\right) \lambda+\mu-\frac{\phi}{(\phi+\mu)}\left(1-w_{1}\right)\right]}, 0,0,0\right)
$$

Based on the mathematical model above, the endemic equilibrium is attained when $S=S^{*}, V=V^{*}, I=I^{*}, T=T^{*}, A=A^{*}$ 
The endemic state is therefore represented by:

$$
\begin{aligned}
\varepsilon_{1}= & \left(\frac{\Lambda}{\left[\left(1+w_{1}\right) \lambda+\psi+\mu-\psi\left(1+w_{1}\right) \lambda\right]}, \frac{\left(1+w_{1}\right) \lambda \Lambda}{[\psi+\mu]\left[\left(1+w_{1}\right) \lambda+\psi+\mu-\psi\left(1+w_{1}\right) \lambda\right]},\right. \\
& \frac{\Lambda \psi}{\left[\left(1+w_{1}\right) \lambda+\psi+\mu-\phi\left(1-w_{1}\right) \lambda\right]\left[\left(1+w_{2}\right)(1-\gamma)+\gamma+\mu\right]}, \\
& \frac{\left[\gamma(\sigma+\mu)+\sigma\left(1+w_{2}\right)(1-\gamma)\right] I^{*}}{(\theta+\alpha+\mu)(\sigma+\mu)-\mu \theta}, \\
& \left.\frac{\left[\left(1+w_{2}\right)(1-\gamma)[(\theta+\alpha+\mu)(\sigma+\mu)-\sigma \theta]+\theta\left(\gamma(\sigma+\mu)+\sigma\left(1+w_{2}\right)(1-\lambda)\right) I^{*}\right]}{[(\theta+\alpha+\mu)(\sigma+\mu)-\sigma \theta][\sigma+\theta]}\right) .
\end{aligned}
$$

Consequently, the basic reproduction number was obtained using the next generation method as;

$$
\begin{aligned}
R_{0}= & \frac{C_{1} \beta_{1}}{\left[\left(1+w_{2}\right)(1-\gamma)+\gamma+\mu\right]} \\
& +\frac{\left[\left(1+w_{2}\right)(1-\gamma)(\theta+\alpha+\mu)+\theta \gamma\right] C_{2} \beta_{2} \mu}{\left[\left(1+w_{2}\right)(1-\gamma)+\gamma+\mu\right][(\sigma+\mu)(\theta+\alpha+\mu)+\theta \sigma]\left[\left(1+w_{1}\right) \lambda+\mu-\phi\left(1+w_{1}\right) \lambda\right]} .
\end{aligned}
$$

\section{Numerical Simulations}

In this section, Matlab software was used to simulate the models. In order to show the analytical results of the work, numerical simulations of the model system are carried out using a set of values.

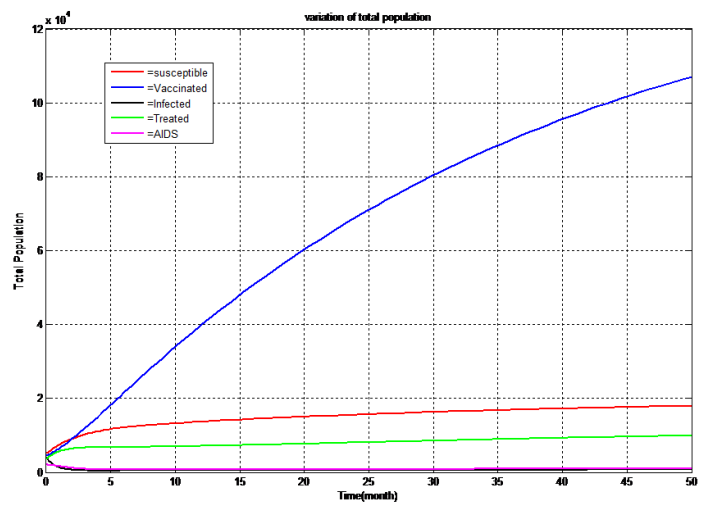

Figure 2. Variation of total population with time

Figure (2) above shows the variation of the proportion of total population in all classes with time using the followng parameters; $\Lambda=5000, w_{1}=0.01, w_{2}=0.25, \lambda=0.35, \gamma=0.40$, $(1-\gamma)=0.60, \sigma=0.061, \phi=0.41, \psi=0.025, \beta_{1}=0.020, \beta_{2}=0.010, C_{1}=0.002, C_{2}=$ $0.025,0.015 \leq \mu \leq 0.025$ and $0.30 \leq \alpha \leq 0.45$

With serious campaign for vaccination and therapy as seen above, the proportion of susceptible increases, there is a significant decrease in the AIDS and infected classes and increase in vaccination class. The treatment class show an increase and finally dropped. 


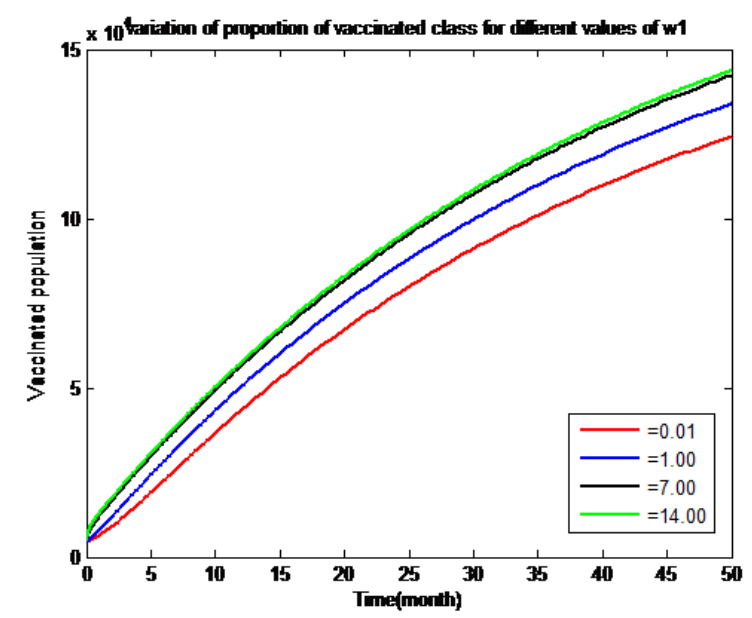

Figure 3. Variation of proportion of vaccinated class for different values of $w_{1}$

Figure (3) above shows the variation of proportion of vaccination class for different value of public health campaign $w_{1}$ with parameter values $\left(w_{1}=0.01,1.00,7.00,14.00\right)$. this shows that increase in the public health campaign will lead to increase in vaccination and many people will be covered, when campaign is vigorously increase, it will reduce stigmatisation of people living with the virus.

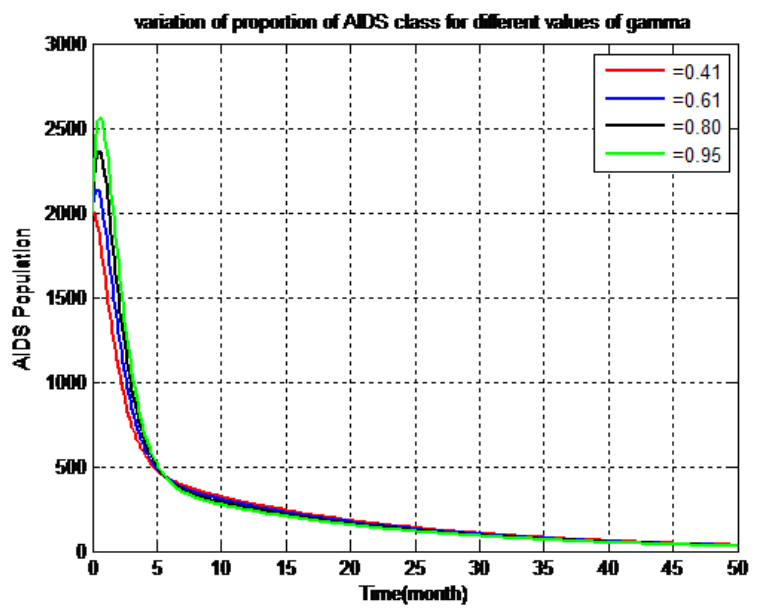

Figure 4. Variation of proportion of AIDS class for different values of $\gamma$

Figure (4) above shows the variation of the proportion of HIV/AIDS class for different values of $\gamma$, showing that the class decrease with increase in $\gamma$, the rate which the infected develop full blown AIDS will drastically reduce.

Figure (5) above shows variation of proportion of treatment class for different values of $\left(w_{2}\right)$, the public health campaign for infectives to receive treatment, the class increase initially, and drop because the population of the infected will drop significantly. 


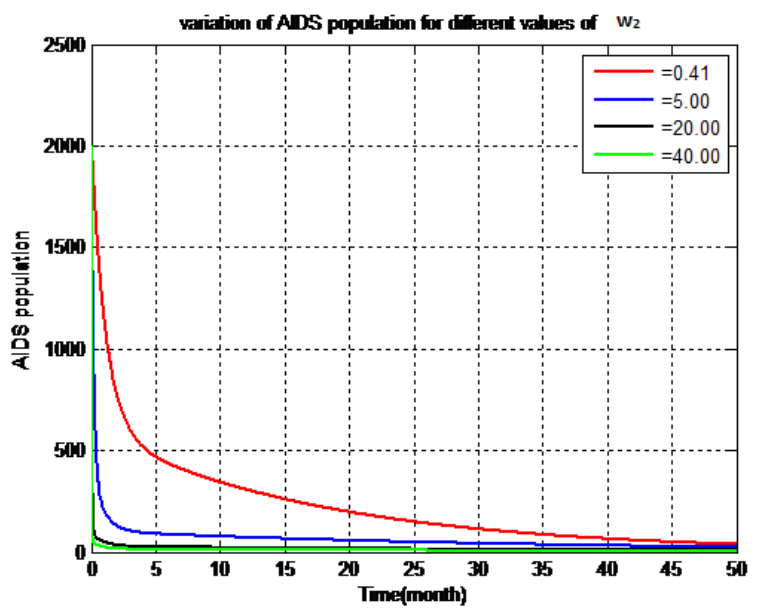

Figure 5. Variation of AIDS population for different values of $w_{2}$

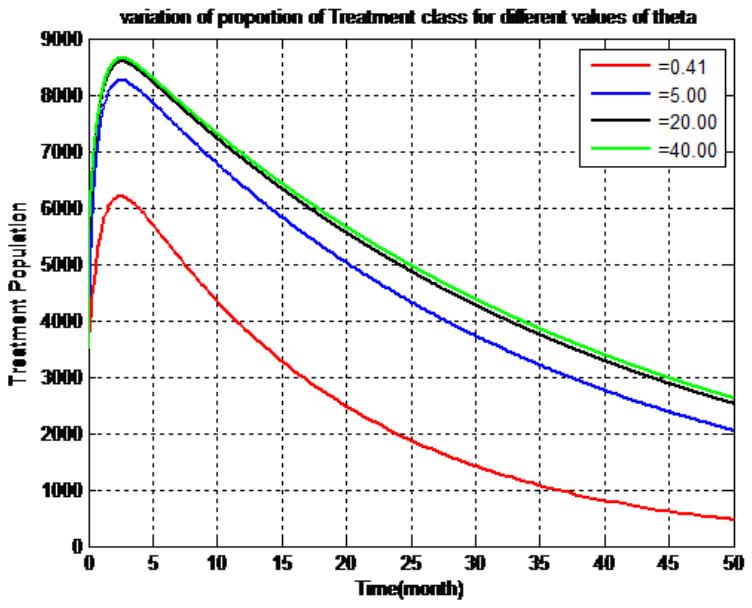

Figure 6. Variation of the population of Treatment class for different values of theta

Figure (6) above shows the variation in the AIDS population for different values of $\theta$. Is is seen that as the $\theta$ increases, the AIDS population decrease and increase the treatment rate.

\section{Discussion and conclusion}

A non-linear mathematical model has been formulated to study the effect of public health campaign on vaccination and treatment for the control of HIV/AIDS within a population. The diseases free equilibrium and endemic equilibrium were obtained and their stabilities investigated. The model showed that the diseases free equilibrium is locally stable when $R_{o}<1$ and unstable when $R_{o}>1$. The basic reproduction number $\left(R_{o}\right)$ was computed using the next generation method. The endemic equilibrium is locally asymptotically stable using the trace 
and determinant method.

A numerical study of the model was carried out to see the effect of public health campaign on the control of the HIV/AIDS virus. The analysis shows that public health campaign on vaccination has the power to reduce the transmission of the diseases and campaign on therapeutic doses has the power to reduce the diseases burden and the transmission of the dreaded virus; by reducing the stigmatisation and consequently reduced the diseases transmission rate. The public education on treatment in every public gathering for those infected with the virus will make those hiding from the public to present themselves for test and treatment which will result in the decline of AIDS diseases/pandemic.

In conclusion, we recommend that the most effective way to reduce the transmission of HIV/AIDS infection is through public health campaign on vaccination when available and screening for individual to know their HIV status, and if an individual is found positive, the person should be educated on the need to willingly undergo ARV treatment and therapy to reduce the viral load and consequently prolonging life of the individual. Education in public place for safe sex should be enhanced and should be encouraged and other preventive measures such as handling unsterilized needle or needle exchange or any activities that may involved exchange of the bloodily fluid containing blood. This means that the national HIV/AIDS programme in most developing countries should keep on encouraging save sex, advocated free counselling and testing and free ARV treatment, if this is maintained the control of HIV/AIDS will be fully achieved within a short time especially within the sub Saharan Africa. HIV/AIDS is much more pronounce in a very poor and undeveloped nation, therefore more advocacy should be enhanced and more centres should be open to allowed free access to treatment and therapy and which will reduce stigmatization on those living with virus therefore reduce the transmission of HIV virus and control the AIDS epidemic all over the world.

\section{References}

[1] A. Kramer, M. Kretzschmar, K. Krickeberg, Modern Infectious Disease Epidemiology (Springer, New York, 2010) 443.

[2] W.N. Rom, S.B. Markowitz, Environmental and Occupational Medicine (Philadelphia : Wolters Kluwer/Lippincott Williams and Wilkins, 2007), 1884.

[3] World Health Organization, HIV/AIDs Fact Sheet. Retrieved from: https://www.who.int/news-room/fact-sheets/detail/hiv-aids (2019 November 15, accessed 2020 January 29).

[4] L.O. Kallings, Journal of internal medicine 263(3), 218-243 (2008).

[5] P.M. Sharp, B.H. Hahn, Cold Spring Harbour Preparative in medicine 1(1):a006841 (2011).

[6] G.E. Gray, F. Laher, E. Lazarus, B. Ensoli, L. Corey, Current opinion in Virology 17, 104-109 (2016).

[7] R.M. Anderson, J. Swinton, G.P. Garnelt, Proceedings. Biological sciences 261(1361), 147-151 (1995).

[8] R.M. Anderson, G.P. Garneth Lancet 348(9033), 1010-1013 (1996).

[9] D.K. Owens, D.M. Edwards, R.D. Shachter, AIDS 12(9), 1057-1066 (1998).

[10] S. Blower, K. Koelle, J. Mills, in Quantitative Evaluation of HIV prevention programs

E. Kaplan and R. Brookmeyer (Eds.) (Yale University Press, New Haven, CT, 2002) 260289. 
[11] R.H. Gray, X. Li, M.J. Wawer, S.J. Gange, D. Serwadda, N.K. Sewankambo, R. Moore, F. Wabwire-Mangen, T. Lutalo, T.C., Rakai Project Group, AIDS 17(13), 1941-1951 (2003).

[12] Z. Mukandavirea, W. Garira, J.M. Tchuencheb, Applied Mathematical Modelling 33(4) 2084-2095 (2009).

[13] A. Gumel, X. W. Zhang, P. N. Shivakumar, M. L. Garba, B. M. Sahai, Journal of Theoretical Medicine 4(2), 147-155 (2002). 